\title{
Trangastric endo-organ resection of a proximal gastric lesion
}

\author{
Piyush Aggarwal $^{1} \cdot$ Kulsoom Laeeq $^{1} \cdot$ Angela Osmolak $^{1} \cdot$ \\ Tommy H. Lee ${ }^{1} \cdot$ Sumeet K. Mittal ${ }^{1}$
}

Received: 11 November 2014 / Accepted: 6 July 2015/Published online: 15 August 2015

(C) Springer Science+Business Media New York 2015

\begin{abstract}
Introduction Gastric tumors confined to mucosa and submucosa can be resected with endoscopic resection techniques. They include endoscopic mucosal resection (EMR) or endoscopic submucosal dissection (ESD) $[1,2]$. These techniques can be challenging when the tumor is large or is near the gastroesophageal (GE) junction. Transgastric resection is a novel technique of removing gastric tumors that are unresectable by endoscopy due to their size and location.

Materials and Methods We present a case of a 41-yearold male where a suspicious appearing lesion near the GE junction was removed using combined trans-gastric laparoscopic and endoscopic technique. The stomach was inflated using endoscopy, and three 5-mm balloon-tipped trocars were inserted directly into the stomach. The lesion was lifted with submucosal injection of saline and was resected using ultrasonic dissection device. The specimen was retrieved using Rothnet through the endoscope. The mucosal defect was closed with absorbable sutures. Trocars were removed and gastrostomy sites were closed with Endostitch device. Swallow study done on post-op day 2 did not show any signs of leak. Patient was discharged
\end{abstract}

home on post-op day 5. Final pathology was consistent with hyperplastic polyp.

Conclusion Proximal Gastric lesions can be safely removed with combined Laparoscopic trans-gastric and endoscopic approach.

Keywords GI $<$ Cancer - Abdominal - Endoscopy · Trans-gastric $\cdot$ Lesion resection $\cdot$ Hybrid technique

Compliance with ethical standards

Disclosures Drs. Piyush Aggarwal, Kulsoom Laeeq, Angela Osmolak, Tommy H. Lee and Sumeet K. Mittal have no conflicts of interest or financial ties to disclose.

\section{References}

1. (2000) Technology status report evaluation. Endoscopic mucosal resection. Gastrointest Endosc 52(6):860-863

2. Oka S, Tanaka S, Kaneko I, Mouri R, Hirata M, Kawamura T, Yoshihara M, Chayama K (2006) Advantage of endoscopic submucosal dissection compared with EMR for early gastric cancer. Gastrointest Endosc 64(6):877-883

Presented at the SAGES 2014 Annual Meeting, April 2-5, 2014, Salt Lake City, Utah.

Electronic supplementary material The online version of this article (doi:10.1007/s00464-015-4413-5) contains supplementary material, which is available to authorized users.

\author{
Piyush Aggarwal \\ drpiyush28@gmail.com \\ 1 Creighton University Medical Center, Omaha, NE, USA
}

